\title{
Fundamental of an efficient and reliable staircase multilevel inverter for photovoltaic application
}

\begin{abstract}
Due to high initial cost of photovoltaic generators, it is necessary to use high efficient converters to convert DC output of Photovoltaic systems to AC. This is done by efficient inverters. Between various inverter structures, staircase output inverters have a special place. Most of these inverters use structures that are constituted of series-connected switches. This limits efficiency and reliability. In this paper, an efficient and reliable staircase output inverter structure based on two ladders of switches is discussed. The major advantages of this structure are higher reliability and efficiency. This structure has low on-state power dissipation which means more performance especially when working with low voltage low power photovoltaic systems. The other advantage of this inverter is reliability when switches fail in form of open circuit. By choosing a special switching strategy, this inverter has ability to continue working with some failed open circuit switches. Increasing the number of levels increases the reliability of this inverter. Moreover, low switching frequency, let to use Photovoltaic output isolator as driver for switches, which means more capability for compactness in Integrated Power Modules (IPM). In this paper, first the structure of this inverter is discussed then the on-state power dissipation is calculated in form of using Mosfets and other $\mathrm{p}-\mathrm{n}$ junction switches, then simulation result is brought.
\end{abstract}

